\title{
Title: Transcriptional regulation of neonatal neural stem cells is a determinant of social behavior
}

\section{Short Title: Neonatal origin of social behavior}

Authors: Takeshi Hiramoto ${ }^{1+}$, Shuken Boku ${ }^{2+}$, Gina Kang ${ }^{1}$, Seiji Abe ${ }^{2}$, Mariel Barbachan e Silva ${ }^{3}$, Kenji Tanigaki ${ }^{4}$, Masako Nagashima², Kenny Ye ${ }^{5}$ Takahira Yamauchi $^{1}$, Tatyana V. Michurina ${ }^{6}$, Pilib Ó Broin ${ }^{3}$, Grigori Enikolopov ${ }^{6,7}$, Noboru Hiroi ${ }^{1,8,9 *}$

\section{Affiliations:}

${ }^{1}$ Department of Pharmacology, University of Texas Health Science Center at San Antonio; TX 78229, USA. ${ }^{2}$ Department of Psychiatry and Behavioral Sciences, Albert Einstein College of Medicine; Bronx, NY 10461, USA.

${ }^{3}$ School of Mathematical \& Statistical Sciences, National University of Ireland Galway; Galway, Ireland.

${ }^{4}$ Research Institute, Shiga Medical Center, 5-4-30 Moriyama, Moriyama-shi, Shiga, Japan.

${ }^{5}$ Department of Epidemiology \& Population Health, Albert Einstein College of Medicine; Bronx, NY 10461, USA.

${ }^{6}$ Center for Developmental Genetics, Stony Brook University, Stony Brook, NY 11794, USA.

${ }^{7}$ Department of Anesthesiology, Stony Brook University; Stony Brook, NY 11794, USA.

${ }^{8}$ Department of Cellular and Integrative Physiology, University of Texas Health Science Center at San Antonio; TX 78229, USA.

${ }^{9}$ Department of Cell Systems and Anatomy, University of Texas Health Science Center at San Antonio; TX 78229, USA.

Present addresses:

SB, Department of Neuropsychiatry, Faculty of Life Sciences, Kumamoto University, Kumamoto, Japan

SA, Department of Hospital Pharmaceutics, School of Pharmacy, Showa University, Tokyo, Japan

TY, Department of Psychiatry, Nara Medical University, Nara, Japan

MN, Department of Palliative Medicine, Teikyo University School of Medicine, Tokyo, Japan

†These two authors contributed equally to this work

*Corresponding author. Email: hiroi@uthscsa.edu 
Abstract: Rare gene variants confer a high level of penetrance to neurodevelopmental disorders, but their developmental origin and cellular substrates remain poorly understood. To address this limitation, we explored the role of $T B X 1$, a gene encoded in a rare copy number variant, in cell and mouse models. Here, we report that neonatal Tbx1 deficiency contributes to defective peripubertal social behavior and impairs the proliferation of neonatal neural stem/progenitor cells. Moreover, TBX1 transcriptionally regulates genes linked to post-embryonic neurogenesis and neurodevelopmental disorders associated with other rare gene variants. Our data indicate a precise time window and cell type through which the social dimension is altered by a gene encoded in a rare CNV and provide a potential common mechanistic basis for a group of neurodevelopmental disorders.

One-Sentence Summary: $T b x 1$, a gene affecting neonatal stem cell proliferation, influences peripubertal social behavior. 


\section{Main Text:}

Identifying the developmental origin of neurodevelopmental disorders and the cell types in which gene variants cause such disorders is essential to develop mechanism-based precision medicine in psychiatry. Rare copy number variants (CNVs) provide a reliable entry point to delve into the pathophysiology of high-penetrance genetic risk factors, linking genes to cellular phenotypes and behavioral consequences. However, CNVs pose conceptual and technical challenges, as each CNV is associated with multiple mental illnesses. A technical challenge is that any CNV might encode many functionally diverse genes. In theory, to determine how CNV-encoded single genes affect neurodevelopmental disorders, isolated dose manipulation of each CNV-encoded single gene (or a set of such genes) and identification of the resulting phenotypes are required. However, such experimental manipulation is not feasible in humans.

As an alternative strategy, CNV-encoded driver genes can be inferred from the protein-truncating variants of each single gene in disease cases. Recent large-scale exome-sequencing studies have identified ultra-rare variants of single genes encoded in CNVs among individuals with schizophrenia and autism spectrum disorder (ASD) $(1,2)$. However, the failure to identify such variants does not necessarily prove their absence. In fact, small-scale exome-sequencing studies have not identified ultra-rare variants that larger scale analyses have. Moreover, ultra-rare variants may also exist in introns and promoter/enhancers of CNV-encoded single genes (3). Further, it is possible that such variants simply do not exist in nature but a one-copy deletion of such single genes within a CNV produces certain

20 phenotypes.

Hemizygous deletion at human chromosome $22 q 11.2$ is associated with elevated rates of schizophrenia and diverse neurodevelopmental disorders, including ASD, intellectual disability, and attention-deficit hyperactivity disorder (4). Ultra-rare cases of protein-truncating variants of TBX1 have also been reported in some families, and some of those carriers have been diagnosed with $\operatorname{ASD}(5,6)$. However, 
these individuals also carry variants of genes outside $22 q 11.2$, precluding the establishment of a causative role for this gene variant (5). A recent large-scale exome-sequencing study of schizophrenia cases identified a protein-truncating variant of TBX1 (1). However, power limitations of these human studies render the contribution of $T B X 1$ to mental illnesses or their dimensions uncertain.

The causal relation between CNVs and phenotypes can also be explored along dimensions crossing clinical boundaries (7). Many dimensions of social and cognitive domains common to ASD and schizophrenia are negatively impacted by $\operatorname{CNVs}(8,9)$. While animal models do not recapitulate clinically defined mental disorders, a dimensional analysis provides an opportunity to translate human phenotypes to those in experimental animals. Complementing the limitations of human studies, reports on genetic mouse models for single CNV-encoded genes have suggested that some 22q11.2-encoded genes contribute to distinct dimensional aspects of mental illnesses (10). Constitutive heterozygosity of Tbx1 in a homogeneous genetic background impairs social communication and interaction in mice (1113). We capitalized on these observations to further identify the precise developmental time window, 15 critical cell type, and functions associated with Tbx1 in mouse and cell models.

\section{RESULTS}




\section{Critical developmental time points of Tbx1 for hippocampal neurogenesis}

Human TBX1 and its murine homolog Tbx1 are expressed in the brains of humans (14) and mice (15), respectively. TBX1 protein levels in the whole mouse brain and hippocampus precipitously declined from the embryonic period to adulthood (Fig. S1A-C). Constitutive Tbx1 heterozygosity alters cortical neurogenesis at embryonic day 13.5 (E13.5) in some, but not all cortical regions $(16,17)$. In the hippocampus of the adult mouse, TBX1 protein is more enriched in the granule cell layer of the hippocampus than in the cortex (11). We examined the rate of embryonic neurogenesis in the hippocampus at E18, as embryonic neurogenesis in the hippocampal granule cell layer peaks around E17-E19 in rodents (18). BrdU injected at E18 labeled indistinguishable numbers of cells in various hippocampal regions in $T b x 1^{+/+}$and $T b x 1^{+/-}$mice (Fig. S2), indicating that $T b x 1$ heterozygosity had no detectable impact on embryonic neurogenesis in the hippocampus.

The granule cell layer of the hippocampus undergoes protracted neurogenesis, up to postnatal day 18 (P18), and a small fraction of neurogenesis persists in the subgranular zone toward adulthood (18). However, the number of BrdU-positive cells in the hippocampus was statistically indistinguishable between $T b x 1^{+/+}$and $T b x 1^{+/-}$mice at P35 (Fig. S3).

Several interpretative issues are associated with $\operatorname{BrdU}(19,20)$. A single dose of $\operatorname{BrdU}$ labels the DNA synthesis of S-phase cells at the time of injection, but it is not a marker of dividing cells per se. While a higher dose or repeated injections of BrdU would label more cells, it could additionally label cell death or repair. Moreover, the number of BrdU-labeled cells could be confounded by altered lengths of the Sphase and total cell cycle. Thus, to reveal the actual neural stem/progenitor cells during the postnatal period, we developed $\operatorname{Tg}\left(\right.$ Nes-EGFP)33Enik/J; Tbx $1^{+/-}$mice (see Supplementary Information) and counted the numbers of GFP-positive neural stem/progenitor cells in the subgranular zone at P35. There were significantly fewer GFP-positive cells in the subgranular zone of the dorsal hippocampus in $\mathrm{Tg}$ (Nes- 


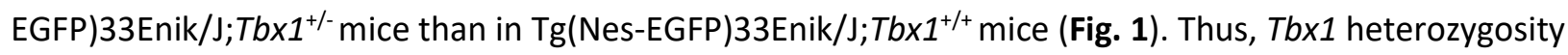
reduces the number of stem/progenitor cells in the postnatal hippocampus.

\section{Cellular consequences of Tbx1 deficiency in neonatal neural stem/progenitor cells}

We further explored the precise manner through which $T b x 1$ heterozygosity impacts the stem/progenitor cell population. To determine the cell-autonomous effect of $T b x 1$ deficiency on the cell cycle of stem/progenitor cells, we cultured and isolated stem/progenitor cells derived from the P0 hippocampus of C57BL/6J pups. This developmental time point was chosen because the TBX1 protein level is higher in the hippocampus earlier than later in the post-embryonic period (see Fig. S1C), and constitutive $T b x 1$ heterozygous mice show defective social communication as early as P7-8 (11-13). Cells were made to simultaneously enter the cell cycle simultaneously using the double-thymidine block method (21). TBX1 protein levels peaked during the G1/S phase shortly before the levels of cyclin B1-a marker of the G2/M phase of the cell cycle-peaked (Fig. 2A).

When we reduced TBX1 expression level using siRNA in vitro (Fig. S1B) and examined the rate of proliferation of neonatal neural stem/progenitor cells under proliferating conditions, Tbx1 siRNA significantly reduced the cell proliferation rate (Fig. 2B). As this is a selective population of neonatal neural stem/progenitor cells under a maximally proliferative condition in vitro, the detection sensitivity of $\mathrm{BrdU}$ is expected to be higher than that of in vivo samples. Tbx1 siRNA reduced the number of BrdUpositive cells (Fig. 2C; Fig. S4). Moreover, the reduced proliferation rate was not due to apoptosis (Fig. 2D). Thus, TBX1 deficiency cell-autonomously reduces the proliferation rate of neonatal hippocampal neural stem/progenitor cells. 


\section{Behavioral consequences of Tbx1 deficiency in neonatal neural stem/progenitor cells}

Constitutive $T b x 1$ heterozygous pups exhibit defective neonatal social communication as early as P7-8

$(11,13)$, and Tbx1 deficiency blunts the proliferation of neural stem/progenitor cells derived from the P0 hippocampus (see Fig. 2B). Therefore, we investigated whether Tbx1 insufficiency during the neonatal period is critical for the normal development of peripubertal social behavior. To initiate Tbx1 heterozygosity in neural stem/progenitor cells at specific post-embryonic periods, we developed

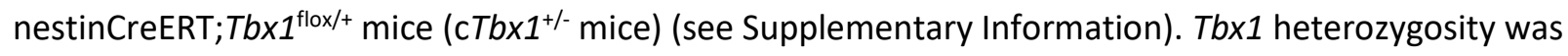
initiated by tamoxifen at P1-5 and mice were behaviorally tested 1 month later (Fig. 3A); it takes at least 1 month for new neurons to be incorporated into the hippocampal circuitry in rodents (22). Recombination was found to be selectively induced in newly generated neurons in brain regions with known post-embryonic neurogenesis in response to tamoxifen (Fig. S6). As vehicle-treated nestinCreERT;Tbx $1^{\text {flox }}$ mice and tamoxifen-treated wild-type;Tbx $1^{\text {flox }}$ mice did not differ in social behavior, they were combined as a single control group (Fig. S5A-E). cTbx $1^{+/-}$mice showed a social approach deficit (Fig. 3B), with normal levels of approach behavior toward a novel object (Fig. 3C), anxiety-related traits (Fig. 3D), and motor behavior (Fig. 3E). The predominant form of social approach was sniffing; therefore, we additionally investigated whether the social approach deficit in $c T b \times 1^{+/-}$mice was due to their abnormal reaction to social olfactory cues. Control and $c T b \times 1^{+/-}$mice were indistinguishable in their responses and habituation to various non-social and social odorants (Fig.

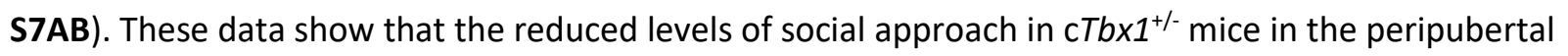
period (i.e., 1 month of age) are not due to the generalized deficits in olfactory responses to social cues, novel object approach, anxiety, or motor capacity.

To further examine the specificity of the neonatal period, we initiated tamoxifen treatment at P21-25 in a different group of mice and tested them 1 month later (i.e., at 2 months of age) (Fig. 3F); mice of various control genotypes did not differ, and they were combined as a single control group (Fig. S5F-J). These $c T b \times 1^{+/-}$mice showed no deficits in social approach, an anxiety-related trait, or motor capacity in an open field (Fig. 3G, I, and J), although they showed impaired ability to approach a novel non-social 
object (Fig. 3H). That tamoxifen treatment at P21-25 affected novel object approach indicates that this treatment per se was effective, ruling out the possibility that the tamoxifen dose was insufficient to impact adult neurogenesis and behavior.

The ablation of postnatal/adult neural stem/progenitor cells at P28 or later has no effect on the subsequent social approach/interaction per se in mice (23-25). Our observations are consistent with these findings in that Tbx1 deletion in stem/progenitor cells at P21-25 had no detectable effect on later social approach/interaction in male mice, but further extend the previous studies by showing that neonatal neurogenesis is more critical for the normal development of social behavior than postnatal neurogenesis and Tbx1 functionally contributes to the development of peripubertal social behavior via its actions during the neonatal period.

\section{Molecular network of TBX1 in neonatal neural stem/progenitor cells}

Having identified neonatal neural stem/progenitor cells as a cellular substrate through which $T b x 1$ deficiency causes social approach deficits later, we next examined the molecular targets of this transcription factor using chromatin immunoprecipitation (ChIP)-seq. We determined TBX1-binding sites under a proliferating condition in neonatal stem/progenitor cells derived from the hippocampus of PO C57BL/6J mice. We ran two sets of ChIP-seq independently using different protocols. TBX1-binding sites were associated mostly with either two genes $(65 \%)$ or one gene $(30 \%)$, and in some instances at sites with no nearby genes (5\%) (Fig. S8A). This analysis collectively identified 1,257 genes and mRNA variants (Table S1-1), comprising 1,039 genes at and near the TBX1-binding sites. The distances between the transcription start site and TBX1 binding sites were most frequently found at 50-500 kb in both directions, followed by 5-50 kb, more than $500 \mathrm{~kb}$, and less than $5 \mathrm{~kb}$ in this order (Fig. S8B). The majority of TBX1-binding sites were intergenic regions (73.42\%), followed by introns (12.12\%), upstream regions (10.55\%), promoters (2.96\%), and exons (0.95\%) (Fig. S9). 
Biological annotations showed the enrichment of G-protein-coupled receptor signaling pathways, epigenetic gene regulation, and chromatin silencing among biological processes (Fig. S10-1); apical junction complex and cell-cell junctions among cellular components (Fig. S10-2); and transcription factor activity, G-protein-coupled activity, structural molecule activity, and transmembrane receptor activity among the molecular functions (Fig. S10-3).

Next, we explored databases of genes relevant to adult hippocampal neurogenesis (MANGO v3.2). It should be cautioned that in the absence of a gene database for neonatal neurogenesis, the MANGO database was used as a proxy. Nonetheless, this search identified 30 TBX1-bound genes that were implicated in adult neurogenesis (Fig. 4A yellow circle; Table S1-2), 22 of which form molecular networks with TBX1 (Fig. 4B). We next asked whether our pool of TBX1-bound genes is involved in ASD (Table S1-3) and developmental brain disorders (Table S1-4). This search identified 87 genes (Fig 4A blue circle). Tbx1 potentially interacts with 61 such genes (Fig. S11), among which 12 genes were also listed in the MANGO database, i.e., Cacna1d, Cnr1, Dcc, Dcx, Disc1, Dmd, Dyrk1a, Foxg1, Gria1, Pten, Tbr1, and Tdo2 (Fig. 4A), rare variants of which are associated with neurodevelopmental disorders, including ASD.

Molecular networks are based on lenient inclusive criteria; the Search Tool for Retrieval of Interacting Genes/Proteins (STRING) database contains any association among genes or molecules as long as they are mentioned in the same articles (26). While such networks are useful for their heuristic value, they may not represent real, functional associations. For example, while a TBX1-PTEN network was identified using the databases of adult neurogenesis (Fig. 4B) and neurodevelopmental disorders (Fig. S11), this association was not based on any functional validation. Thus, we performed a series of validations. Our 
colocalized with PTEN in neonatal neural stem/progenitor cells derived from the PO hippocampus of C57BL/6J pups (Fig. 5A, left) and in a subpopulation of PTEN-positive cells in the hippocampal granular zone of C57BL/6J mice (Fig. 5A, right). The Pten promoter was enriched in DNA fragments precipitated by a TBX1 antibody, as determined by ChIP-PCR (Fig. 5B). Tbx1 siRNA significantly reduced Pten promoter activity (Fig. 5C) and PTEN protein levels (Fig. 5D). TBX1 protein was found to be bound 315 bp upstream and 84,805 downstream of the Pten transcription start site (Fig. S12A-B). The former site is within a reasonable enhancer location distance (27). However, the binding locations of transcription factors revealed by ChIP-seq are not reliable in terms of the precise distances from the target genes owing to the size of fragments generated by sonication (28). Moreover, the abundance of binding may not necessarily indicate functionally critical loci. Thus, we constructed various promoter sequences of Pten with deletions and mutations at different loci (Fig. 5E, left) and examined their transcriptional activity. While the Pten promoter is constitutively active, its transcriptional activity doubles during proliferation with epidermal growth factor (EGF) (see Fig. S13). The presence of the full-length Pten promoter was essential for luciferase activity (Fig. S14). The transcriptional activation of the Pten promoter under this condition was reduced by a deletion or mutation placed at the proximal $35 \mathrm{bp}$ segment (Fig. 5E, right), which contains a recognition sequence resembling the T-box consensus sequence (29). Our observations that $T b x 1$ deficiency results in a blunted increase in the number of neonatal hippocampal neural stem/progenitor cells (see Fig. 2B) and reduction in the transcriptional activation and expression levels of PTEN (see Fig. 5C-D) are consistent with the eventual depletion of stem/progenitor cells in the adult hippocampal granule cell layer following Pten deletion in vivo (30).

Molecular networks identified by STRING uncovered Flt1, ApoE, and Tdo2 as potential indirect interacting molecules that are also implicated in adult neurogenesis (Fig. 4AB). While TBX1-binding sites are outside a 2 kb promoter region (Table S1-1), Flt1 was identified in four separate clones of ChIP-seq 25 and thus included. ApoE has not been implicated in neurodevelopmental disorders, but its indirect link with TBX1 via PTEN is a possibility (see Fig. 4B). Tryptophan 2,3-dioxygenase (TDO2) is the rate-limiting 
enzyme in the catabolism of tryptophan in the kynurenine pathway, the downregulation of which has been implicated in ASD (31). We additionally tested Btg1, as it was identified in MANGO (Table S1-2) but not in databases of neurodevelopmental disorders (see Table S1-3; S1-4). We reduced Tbx1 mRNA expression levels in the culture of neonatal neural stem/progenitor cells using $T b x 1$ siRNA. This analysis showed that $T b x 1$ siRNA reduced $T b x 1$ mRNA by half (Fig. S15A) and reduced Tdo2 mRNA levels but did not affect levels of Flt1, ApoE, or Btg1 mRNA (Fig. S15B-E). Tdo2 mRNA is also highly enriched in the hippocampal granule cell layer in the mouse brain (32) and is critical for the proliferation of adult neural stem/progenitor cells (33).

TBX1 regulates the proliferation of neonatal neural stem/progenitor cells (see Fig. 2B), and Pten deletion leads to a transitory expansion of hippocampal adult stem/progenitor cells and other stem cells, followed by the exhaustion of the precursor pool, presumably through an imbalanced increase in the extent of $\mathrm{G} 0$ to $\mathrm{G} 1$ transition $(34,35)$. Therefore, we further explored the potential target genes implicated in the G0/G1 phase among ChIPseq-identified TBX1-bound genes, which revealed Ecrg4 and Rnf112 (Table S1-1; Table S2). TBX1-binding sites were found near Ecrg4 and Rnf112 (Fig. S17-S18;

Table S1-1). Tbx1 siRNA significantly reduced the expression of Ecrg4 and Rnf112 mRNA (Fig. S15F-G). No functional roles of these genes in neonatal neural stem/progenitor cells in the hippocampus are known. However, ECRG4 suppresses the proliferation of stem cells in the mouse dentate gyrus (36). Our data identify potential molecular targets whereby TBX1 regulates the cell cycle in hippocampal neonatal neural stem/progenitor cells. 


\section{DISCUSSION}

We established that neonatal $T b x 1$ deficiency impairs peripubertal social behavior and impairs the proliferation of neonatal neural stem/progenitor cells. Therefore, our study provides a potential cellular basis for a social dimension of neurodevelopmental disorders linked to TBX1 variants and a critical developmental time point at which TBX1 determines the level of a subsequent social dimension. This gene transcriptionally acts on 87 genes that are independently associated with neurodevelopmental disorders; 12 of these genes are also known to be involved in adult neurogenesis. Interestingly, 75 of the 87 genes associated with neurodevelopmental disorders have not been implicated in adult or neonatal neurogenesis. Moreover, 18 TBX1-bound genes that are listed in the adult neurogenesis database have not been known to be associated with neurodevelopmental disorders. This pool of genes provides a means to identify novel genes contributing to the social dimension of neurodevelopmental disorders via neonatal neurogenesis. Thus, our data link TBX1, which has its own ultra-rare gene variants and is also encoded in a rare CNV, to a set of other genes, rare variants of which are associated with neurodevelopmental disorders.

How CNV-encoded genes and their biological processes contribute to various psychiatric disorders is still poorly understood. The identification of driver genes within CNVs in humans is limited due to the very rare nature of variants of their encoded genes. Recent large-scale exome sequencing studies have identified more ultra-rare variants $(1,37)$, but these data do not necessarily rule out the possibility that dose alterations--not their own variants-of CNV-encoded genes contribute to phenotypes. Moreover, while embryonic neurogenesis, synaptic functions, and activity-regulated cytoskeleton-associated proteins have emerged as common, robust candidate processes affecting mental illnesses, such analyses are not devoid of technical and interpretative limitations (38). Literature-curated databases do not provide genes and biological processes whose functions have not been explored or are not abundantly or commonly represented. 
Our approach, starting from a CNV-encoded gene, represents a complementary strategy to circumvent the limitations associated with human studies and to identify driver genes, and their cell functions and dimensional behavioral phenotypes. We capitalized on TBX1, a 22q11.2 CNV-encoded gene, of which several cases of ultra-rare variants exist with variable neurodevelopmental disorders $(1,5,6,39,40)$.

The causative roles of TBX1 in phenotypes have not been established due to their lack of statistical power (1) and the presence of other variants among carriers (5) in humans. Given that the expression of TBX1 protein is enriched in the sites of post-embryonic neurogenesis (11), we focused on the function of this gene in post-embryonic stem cells in mice. This approach provided a theoretical basis for grouping dimensional aspects of neurodevelopmental disorders, based on a shared mechanistic basis, a prerequisite for designing mechanism-based precision medicine to ultimately treat specific dimensions of such disorders in humans (7). 


\section{References and Notes:}

1. T. Singh, B.M. Neale, M.J Daly, SCHEMA, Exome sequencing identifies rare coding variants in 10 genes which confer substantial risk for schizophrenia. MedRxiv, (2020).

2. F. K. Satterstrom, J. A. Kosmicki, J. Wang, M. S. Breen, S. De Rubeis, J. Y. An, M. Peng, R. Collins, J. Grove, L. Klei, C. Stevens, J. Reichert, M. S. Mulhern, M. Artomov, S. Gerges, B. Sheppard, X. Xu, A. Bhaduri, U. Norman, H. Brand, G. Schwartz, R. Nguyen, E. E. Guerrero, C. Dias, Consortium Autism Sequencing, Psych-Broad Consortium i, C. Betancur, E. H. Cook, L. Gallagher, M. Gill, J. S. Sutcliffe, A. Thurm, M. E. Zwick, A. D. Borglum, M. W. State, A. E. Cicek, M. E. Talkowski, D. J. Cutler, B. Devlin, S. J. Sanders, K. Roeder, M. J. Daly, J. D. Buxbaum, Large-Scale Exome Sequencing Study Implicates Both Developmental and Functional Changes in the Neurobiology of Autism. Cell 180, 568584 e523 (2020).

3. I. Mitra, B. Huang, N. Mousavi, N. Ma, M. Lamkin, R. Yanicky, S. Shleizer-Burko, K. E. Lohmueller, M. Gymrek, Patterns of de novo tandem repeat mutations and their role in autism. Nature 589, 246-250 (2021).

4. J. Zinkstok, E. Boot, A.S. Bassett, N. Hiroi, N.J. Butcher, C. Vingerhoets, J.A.S. Vorstman, T.A.M.J. van Amelsvoort, The 22q11.2 deletion syndrome from a neurobiological perspective. Lancet Psychiatry 6, 951-960. (2019).

5. T. Ogata, T. Niihori, N. Tanaka, M. Kawai, T. Nagashima, R. Funayama, K. Nakayama, S. Nakashima, F. Kato, M. Fukami, Y. Aoki, Y. Matsubara, TBX1 mutation identified by exome sequencing in a Japanese family with 22q11.2 deletion syndrome-like craniofacial features and hypocalcemia. PLoS. One 9, e91598 (2014).

6. R. Paylor, B. Glaser, A. Mupo, P. Ataliotis, C. Spencer, A. Sobotka, C. Sparks, C.H. Choi, J. Oghalai, S. Curran, K.C. Murphy, S. Monks, N. Williams, M.C. O'Donovan, M.J. Owen, P.J. 
Scambler, E. Lindsay, Tbx1 haploinsufficiency is linked to behavioral disorders in mice and humans: implications for $22 q 11$ deletion syndrome. Proc. Natl. Acad. Sci. U. S. A 103, 7729-7734 (2006).

7. T. R. Insel, B. N. Cuthbert, Medicine. Brain disorders? Precisely. Science 348, 499-500 (2015).

8. R.E. Gur, J.J. Yi, D.M. Donald-McGinn, S.X. Tang, M.E. Calkins, D. Whinna, M.C. Souders, A. Savitt, E.H. Zackai, P.J. Moberg, B.S. Emanuel, R.C. Gur, Neurocognitive development in 22q11.2 deletion syndrome: comparison with youth having developmental delay and medical comorbidities. Mol. Psychiatry 19, 1205-1211 (2014).

9. H. Stefansson, A. Meyer-Lindenberg, S. Steinberg, B. Magnusdottir, K. Morgen, S. Arnarsdottir, G. Bjornsdottir, G. B. Walters, G. A. Jonsdottir, O. M. Doyle, H. Tost, O. Grimm, S. Kristjansdottir, H. Snorrason, S. R. Davidsdottir, L. J. Gudmundsson, G. F. Jonsson, B. Stefansdottir, I. Helgadottir, M. Haraldsson, B. Jonsdottir, J. H. Thygesen, A. J. Schwarz, M. Didriksen, T. B. Stensbol, M. Brammer, S. Kapur, J. G. Halldorsson, S. Hreidarsson, E. Saemundsen, E. Sigurdsson, K. Stefansson, CNVs conferring risk of autism or schizophrenia affect cognition in controls. Nature 505, 361-366 (2014).

10. N. Hiroi, T. Yamauchi, Modeling and Predicting Developmental Trajectories of Neuropsychiatric Dimensions Associated With Copy Number Variations. Int J Neuropsychopharmacol 22, 488-500 (2019).

11. T. Hiramoto, G. Kang, G. Suzuki, Y. Satoh, R. Kucherlapati, Y. Watanabe, N. Hiroi, Tbx1: identification of a $22 q 11.2$ gene as a risk factor for autism spectrum disorder in a mouse model. Hum Mol Genet 20, 4775-4785 (2011).

12. R. Kato, A. Machida, K. Nomoto, G. Kang, T. Hiramoto, K. Tanigaki, K. Mogi, N. Hiroi, T. Kikusui, Maternal approach behaviors toward neonatal calls are impaired by mother's 
experiences of raising pups with a risk gene variant for autism. Dev Psychobiol 63, 108113 (2021).

13. T. Takahashi, S. Okabe, P. O. Broin, A. Nishi, K. Ye, M. V. Beckert, T. Izumi, A. Machida, G. Kang, S. Abe, J. L. Pena, A. Golden, T. Kikusui, N. Hiroi, Structure and function of neonatal social communication in a genetic mouse model of autism. Mol Psychiatry 21, 1208-1214 (2016).

14. M. J. Hawrylycz, E. S. Lein, A. L. Guillozet-Bongaarts, E. H. Shen, L. Ng, J. A. Miller, L. N. van de Lagemaat, K. A. Smith, A. Ebbert, Z. L. Riley, C. Abajian, C. F. Beckmann, A. Bernard, D. Bertagnolli, A. F. Boe, P. M. Cartagena, M. M. Chakravarty, M. Chapin, J. Chong, R. A. Dalley, B. David Daly, C. Dang, S. Datta, N. Dee, T. A. Dolbeare, V. Faber, D. Feng, D. R. Fowler, J. Goldy, B. W. Gregor, Z. Haradon, D. R. Haynor, J. G. Hohmann, S. Horvath, R. E. Howard, A. Jeromin, J. M. Jochim, M. Kinnunen, C. Lau, E. T. Lazarz, C. Lee, T. A. Lemon, L. Li, Y. Li, J. A. Morris, C. C. Overly, P. D. Parker, S. E. Parry, M. Reding, J. J. Royall, J. Schulkin, P. A. Sequeira, C. R. Slaughterbeck, S. C. Smith, A. J. Sodt, S. M. Sunkin, B. E. Swanson, M. P. Vawter, D. Williams, P. Wohnoutka, H. R. Zielke, D. H. Geschwind, P. R. Hof, S. M. Smith, C. Koch, S. G. N. Grant, A. R. Jones, An anatomically comprehensive atlas of the adult human brain transcriptome. Nature 489, 391-399 (2012).

15. D.W. Meechan, T.M. Maynard, Y. Wu, D. Gopalakrishna, J.A. Lieberman, A.S. LaMantia, Gene dosage in the developing and adult brain in a mouse model of 22q11 deletion syndrome. Mol. Cell Neurosci 33, 412-428 (2006).

16. G. Flore, S. Cioffi, M. Bilio, E. Illingworth, Cortical Development Requires Mesodermal Expression of Tbx1, a Gene Haploinsufficient in 22q11.2 Deletion Syndrome. Cereb Cortex 27, 2210-2225 (2017). 
17. D. W. Meechan, E. S. Tucker, T. M. Maynard, A. S. LaMantia, Diminished dosage of 22q11 genes disrupts neurogenesis and cortical development in a mouse model of 22q11 deletion/DiGeorge syndrome. Proc Natl Acad Sci U S A 106, 16434-16445 (2009).

18. S.A. Bayer, Development of the hippocampal region in the rat. I. Neurogenesis examined with 3H-thymidine autoradiography. J. Comp Neurol 190, 87-114 (1980).

19. A.J. Eisch, C.D. Mandyam, Adult neurogenesis: can analysis of cell cycle proteins move us "Beyond BrdU"? Curr. Pharm. Biotechnol 8, 147-165 (2007).

20. P. Taupin, BrdU immunohistochemistry for studying adult neurogenesis: paradigms, pitfalls, limitations, and validation. Brain Res Rev 53, 198-214 (2007).

21. C. J. Bostock, D. M. Prescott, J. B. Kirkpatrick, An evaluation of the double thymidine block for synchronizing mammalian cells at the G1-S border. Exp Cell Res 68, 163-168 (1971).

22. C. A. Denny, N. S. Burghardt, D. M. Schachter, R. Hen, M. R. Drew, 4- to 6-week-old adult-born hippocampal neurons influence novelty-evoked exploration and contextual fear conditioning. Hippocampus 22, 1188-1201 (2012).

23. G. S. Kirshenbaum, S. R. Lieberman, T. J. Briner, E. D. Leonardo, A. Dranovsky, Adolescent but not adult-born neurons are critical for susceptibility to chronic social defeat. Front Behav Neurosci 8, 289 (2014).

24. L. Garrett, J. Zhang, A. Zimprich, K. M. Niedermeier, H. Fuchs, V. Gailus-Durner, M. Hrabe de Angelis, D. Vogt Weisenhorn, W. Wurst, S. M. Holter, Conditional Reduction of Adult Born Doublecortin-Positive Neurons Reversibly Impairs Selective Behaviors. Front Behav Neurosci 9, 302 (2015).

25. A. R. Pereira-Caixeta, L. O. Guarnieri, D. C. Medeiros, Emam Mendes, L. C. D. Ladeira, M. T. Pereira, M. F. D. Moraes, G. S. Pereira, Inhibiting constitutive neurogenesis 
compromises long-term social recognition memory. Neurobiol Learn Mem 155, 92-103 (2018).

26. C. von Mering, L. J. Jensen, B. Snel, S. D. Hooper, M. Krupp, M. Foglierini, N. Jouffre, M. A. Huynen, P. Bork, STRING: known and predicted protein-protein associations, integrated and transferred across organisms. Nucleic Acids Res 33, D433-437 (2005).

27. M. Gasperini, A. J. Hill, J. L. McFaline-Figueroa, B. Martin, S. Kim, M. D. Zhang, D. Jackson, A. Leith, J. Schreiber, W. S. Noble, C. Trapnell, N. Ahituv, J. Shendure, A Genome-wide Framework for Mapping Gene Regulation via Cellular Genetic Screens. Cell 176, 377-390 e319 (2019).

28. S. M. Lloyd, X. Bao, Pinpointing the Genomic Localizations of Chromatin-Associated Proteins: The Yesterday, Today, and Tomorrow of ChIP-seq. Curr Protoc Cell Biol 84, e89 (2019).

29. G. Broitman-Maduro, M. Owraghi, W. W. Hung, S. Kuntz, P. W. Sternberg, M. F. Maduro, The NK-2 class homeodomain factor CEH-51 and the T-box factor TBX-35 have overlapping function in C. elegans mesoderm development. Development 136, 27352746 (2009).

30. A. Amiri, W. Cho, J. Zhou, S.G. Birnbaum, C.M. Sinton, R.M. McKay, L.F. Parada, Pten deletion in adult hippocampal neural stem/progenitor cells causes cellular abnormalities and alters neurogenesis. J. Neurosci 32, 5880-5890 (2012).

31. R. Savino, M. Carotenuto, A. N. Polito, S. Di Noia, M. Albenzio, A. Scarinci, A. Ambrosi, F. Sessa, N. Tartaglia, G. Messina, Analyzing the Potential Biological Determinants of Autism Spectrum Disorder: From Neuroinflammation to the Kynurenine Pathway. Brain Sci 10, (2020). 
32. E. S. Lein, M. J. Hawrylycz, N. Ao, M. Ayres, A. Bensinger, A. Bernard, A. F. Boe, M. S.

Boguski, K. S. Brockway, E. J. Byrnes, L. Chen, L. Chen, T. M. Chen, M. C. Chin, J. Chong,

B. E. Crook, A. Czaplinska, C. N. Dang, S. Datta, N. R. Dee, A. L. Desaki, T. Desta, E. Diep,

T. A. Dolbeare, M. J. Donelan, H. W. Dong, J. G. Dougherty, B. J. Duncan, A. J. Ebbert, G.

Eichele, L. K. Estin, C. Faber, B. A. Facer, R. Fields, S. R. Fischer, T. P. Fliss, C. Frensley, S.

N. Gates, K. J. Glattfelder, K. R. Halverson, M. R. Hart, J. G. Hohmann, M. P. Howell, D. P.

Jeung, R. A. Johnson, P. T. Karr, R. Kawal, J. M. Kidney, R. H. Knapik, C. L. Kuan, J. H. Lake,

A. R. Laramee, K. D. Larsen, C. Lau, T. A. Lemon, A. J. Liang, Y. Liu, L. T. Luong, J.

Michaels, J. J. Morgan, R. J. Morgan, M. T. Mortrud, N. F. Mosqueda, L. L. Ng, R. Ng, G. J.

Orta, C. C. Overly, T. H. Pak, S. E. Parry, S. D. Pathak, O. C. Pearson, R. B. Puchalski, Z. L.

Riley, H. R. Rockett, S. A. Rowland, J. J. Royall, M. J. Ruiz, N. R. Sarno, K. Schaffnit, N. V.

Shapovalova, T. Sivisay, C. R. Slaughterbeck, S. C. Smith, K. A. Smith, B. I. Smith, A. J.

Sodt, N. N. Stewart, K. R. Stumpf, S. M. Sunkin, M. Sutram, A. Tam, C. D. Teemer, C.

Thaller, C. L. Thompson, L. R. Varnam, A. Visel, R. M. Whitlock, P. E. Wohnoutka, C. K.

Wolkey, V. Y. Wong, M. Wood, M. B. Yaylaoglu, R. C. Young, B. L. Youngstrom, X. F. Yuan,

B. Zhang, T. A. Zwingman, A. R. Jones, Genome-wide atlas of gene expression in the adult mouse brain. Nature 445, 168-176 (2007).

33. M. Kanai, H. Funakoshi, H. Takahashi, T. Hayakawa, S. Mizuno, K. Matsumoto, T. Nakamura, Tryptophan 2,3-dioxygenase is a key modulator of physiological neurogenesis and anxiety-related behavior in mice. Mol Brain 2, 8 (2009).

34. O. H. Yilmaz, R. Valdez, B. K. Theisen, W. Guo, D. O. Ferguson, H. Wu, S. J. Morrison, Pten dependence distinguishes haematopoietic stem cells from leukaemia-initiating cells. Nature 441, 475-482 (2006). 
35. J. Zhang, J. C. Grindley, T. Yin, S. Jayasinghe, X. C. He, J. T. Ross, J. S. Haug, D. Rupp, K. S. Porter-Westpfahl, L. M. Wiedemann, H. Wu, L. Li, PTEN maintains haematopoietic stem cells and acts in lineage choice and leukaemia prevention. Nature 441, 518-522 (2006).

36. Y. Nakatani, H. Kiyonari, T. Kondo, Ecrg4 deficiency extends the replicative capacity of neural stem cells in a Foxg1-dependent manner. Development 146, (2019).

37. F. K. Satterstrom, R. K. Walters, T. Singh, E. M. Wigdor, F. Lescai, D. Demontis, J. A. Kosmicki, J. Grove, C. Stevens, J. Bybjerg-Grauholm, M. Baekvad-Hansen, D. S. Palmer, J. B. Maller, Psych-Broad Consortium i, M. Nordentoft, O. Mors, E. B. Robinson, D. M. Hougaard, T. M. Werge, P. Bo Mortensen, B. M. Neale, A. D. Borglum, M. J. Daly, Autism spectrum disorder and attention deficit hyperactivity disorder have a similar burden of rare protein-truncating variants. Nat Neurosci 22, 1961-1965 (2019).

38. P. F. Sullivan, D. H. Geschwind, Defining the Genetic, Genomic, Cellular, and Diagnostic Architectures of Psychiatric Disorders. Cell 177, 162-183 (2019).

39. W. Gong, S. Gottlieb, J. Collins, A. Blescia, H. Dietz, E. Goldmuntz, D.M. Donald-McGinn, E.H. Zackai, B.S. Emanuel, D.A. Driscoll, M.L. Budarf, Mutation analysis of TBX1 in nondeleted patients with features of DGS/VCFS or isolated cardiovascular defects. J. Med. Genet 38, E45 (2001).

40. K. Hasegawa, H. Tanaka, Y. Higuchi, Y. Hayashi, K. Kobayashi, H. Tsukahara, Novel heterozygous mutation in TBX1 in an infant with hypocalcemic seizures. Clin Pediatr Endocrinol 27, 159-164 (2018).

41. D. Szklarczyk, A. L. Gable, D. Lyon, A. Junge, S. Wyder, J. Huerta-Cepas, M. Simonovic, N. T. Doncheva, J. H. Morris, P. Bork, L. J. Jensen, C. V. Mering, STRING v11: protein-protein association networks with increased coverage, supporting functional discovery in genome-wide experimental datasets. Nucleic Acids Res 47, D607-D613 (2019). 
42. A. Sebe-Pedros, A. Ariza-Cosano, M. T. Weirauch, S. Leininger, A. Yang, G. Torruella, M. Adamski, M. Adamska, T. R. Hughes, J. L. Gomez-Skarmeta, I. Ruiz-Trillo, Early evolution of the T-box transcription factor family. Proc Natl Acad Sci U S A 110, 16050-16055 (2013).

43. J.L. Mignone, V. Kukekov, A.S. Chiang, D. Steindler, G. Enikolopov, Neural stem and progenitor cells in nestin-GFP transgenic mice. J. Comp Neurol 469, 311-324 (2004).

44. D.C. Lagace, M.C. Whitman, M.A. Noonan, J.L. Ables, N.A. DeCarolis, A.A. Arguello, M.H. Donovan, S.J. Fischer, L.A. Farnbauch, R.D. Beech, R.J. DiLeone, C.A. Greer, C.D. Mandyam, A.J. Eisch, Dynamic contribution of nestin-expressing stem cells to adult neurogenesis. J. Neurosci 27, 12623-12629 (2007).

45. J.S. Arnold, E.M. Braunstein, T. Ohyama, A.K. Groves, J.C. Adams, M.C. Brown, B.E. Morrow, Tissue-specific roles of Tbx1 in the development of the outer, middle and inner ear, defective in 22q11DS patients. Hum. Mol. Genet 15, 1629-1639 (2006).

46. J. Battiste, A.W. Helms, E.J. Kim, T.K. Savage, D.C. Lagace, C.D. Mandyam, A.J. Eisch, G. Miyoshi, J.E. Johnson, Ascl1 defines sequentially generated lineage-restricted neuronal and oligodendrocyte precursor cells in the spinal cord. Development 134, 285-293 (2007).

47. D. Petrik, S. Yun, S. E. Latchney, S. Kamrudin, J. A. LeBlanc, J. A. Bibb, A. J. Eisch, Early postnatal in vivo gliogenesis from nestin-lineage progenitors requires cdk5. PLoS One 8, e72819 (2013)

48. A. M. Ceasrine, N. Ruiz-Otero, E. E. Lin, D. N. Lumelsky, E. D. Boehm, R. Kuruvilla, Tamoxifen Improves Glucose Tolerance in a Delivery-, Sex-, and Strain-Dependent Manner in Mice. Endocrinology 160, 782-790 (2019). 
49. M.Y. Sun, M.J. Yetman, T.C. Lee, Y. Chen, J.L. Jankowsky, Specificity and efficiency of reporter expression in adult neural progenitors vary substantially among nestinCreER(T2) lines. J. Comp Neurol 522, 1191-1208 (2014).

50. S. Boku, T. Izumi, S. Abe, T. Takahashi, A. Nishi, H. Nomaru, Y. Naka, G. Kang, M. Nagashima, A. Hishimoto, S. Enomoto, G. Duran-Torres, K. Tanigaki, J. Zhang, K. Ye, S. Kato, P.T. Mannisto, K. Kobayashi, N. Hiroi, Copy number elevation of 22q11.2 genes arrests the developmental maturation of working memory capacity and adult neurogenesis. Molecular Psychiatry 23, 985-992 (2018).

51. M. Toritsuka, S. Kimoto, K. Muraki, M.A. Landek-Salgado, A. Yoshida, N. Yamamoto, Y. Horiuchi, H. Hiyama, K. Tajinda, N. Keni, E. Illingworth, T. Iwamoto, T. Kishimoto, A. Sawa, K. Tanigaki, Deficits in microRNA-mediated Cxcr4/Cxcl12 signaling in neurodevelopmental deficits in a 22q11 deletion syndrome mouse model. Proc. Natl. Acad. Sci. U. S. A 110, 17552-17557 (2013).

52. H. A. Cameron, R. D. Mckay, Adult neurogenesis produces a large pool of new granule cells in the dentate gyrus. J Comp Neurol 435, 406-417 (2001).

53. M. Nakamura, K. Ye, M. Barbachan E Silva, T. Yamauchi, D. Hoeppner, A. Fayyazuddin, G. Kang, E. Yuda, M. Nagashima, S. Enomoto, T. Hiramoto, R. Sharp, I. Kaneko, K. Tajinda, M. Adachi, T. Mihara, S. Tokuno, M. Geyer, P. O’Broin, M. Matsumoto, N. Hiroi, Computational identification of variables in neonatal vocalizations predictive for postpubertal social behaviors in a mouse model of 16p11.2 deletion. Molecular Psychiatry Online Published on April 15, 2021, (2021).

54. C. Y. McLean, D. Bristor, M. Hiller, S. L. Clarke, B. T. Schaar, C. B. Lowe, A. M. Wenger, G. Bejerano, GREAT improves functional interpretation of cis-regulatory regions. Nat Biotechnol 28, 495-501 (2010). 
55. W. J. Kent, C. W. Sugnet, T. S. Furey, K. M. Roskin, T. H. Pringle, A. M. Zahler, D. Haussler, The human genome browser at UCSC. Genome Res 12, 996-1006 (2002).

56. I. V. Kulakovskiy, I. E. Vorontsov, I. S. Yevshin, R. N. Sharipov, A. D. Fedorova, E. I. Rumynskiy, Y. A. Medvedeva, A. Magana-Mora, V. B. Bajic, D. A. Papatsenko, F. A. Kolpakov, V. J. Makeev, HOCOMOCO: towards a complete collection of transcription factor binding models for human and mouse via large-scale ChIP-Seq analysis. Nucleic Acids Res 46, D252-D259 (2018).

57. B. Rhead, D. Karolchik, R. M. Kuhn, A. S. Hinrichs, A. S. Zweig, P. A. Fujita, M. Diekhans, K. E. Smith, K. R. Rosenbloom, B. J. Raney, A. Pohl, M. Pheasant, L. R. Meyer, K. Learned, F. Hsu, J. Hillman-Jackson, R. A. Harte, B. Giardine, T. R. Dreszer, H. Clawson, G. P. Barber, D. Haussler, W. J. Kent, The UCSC Genome Browser database: update 2010. Nucleic Acids Res 38, D613-619 (2010).

58. G. Yu, L. G. Wang, Q. Y. He, ChIPseeker: an R/Bioconductor package for ChIP peak annotation, comparison and visualization. Bioinformatics 31, 2382-2383 (2015). and Bioconductor: a powerful link between biological databases and microarray data analysis. Bioinformatics 21, 3439-3440 (2005).

60. Richard lannone. (2020), pp. https://rich-iannone.github.io/DiagrammeR/. 
Acknowledgments: We thank Dr. Bernice Morrow for providing the original breeders of $T b x I^{+/-}$ and $T b x 1^{\text {flox/+ }}$ mice.

\section{Funding:}

National Institutes of Health grant R01MH099660 (NH)

National Institutes of Health grant R01DC015776 (NH)

National Institutes of Health grant R21HD105287 (NH)

Fellowship from the Uehara Memorial Foundation (SB)

Fellowship from the Senshin Medical Research Foundation (SB) official views of the National Institute of Health.

\section{Author Contributions:}

Conceptualization: $\mathrm{TH}, \mathrm{NH}$

Investigation: $\mathrm{TH}, \mathrm{NH}, \mathrm{BS}, \mathrm{GK}, \mathrm{SA}, \mathrm{KT}, \mathrm{MN}, \mathrm{MBS}, \mathrm{PÓ}, \mathrm{KY}$,

Visualization: KY, TY

Funding acquisition: $\mathrm{NH}, \mathrm{SB}$

Project administration: $\mathrm{NH}$ 
Writing - review \& editing: $\mathrm{TH}, \mathrm{SB}, \mathrm{NH}$

Competing interests: Authors declare that they have no competing interests.

Data and material availability: All data are deposited at https://datadryad.org/stash/. Mice are available through material transfer agreement.

\title{
Supplementary Materials:
}

Materials and methods

\author{
Table S1-S5
}

Fig S1-S15

References (41-60) 


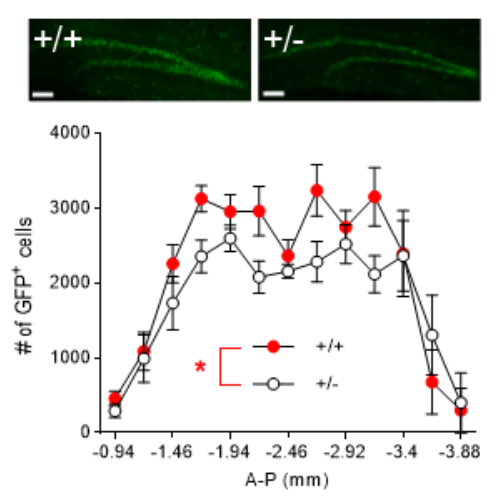

Fig. 1. Effect of constitutive Tbx1 heterozygosity on adult neural stem/progenitor cells in the mouse

hippocampus. (A) Representative images of GFP-positive cells in the subgranular zone of the

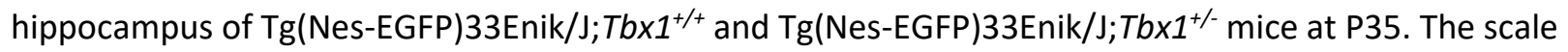
bar indicates $100 \mu \mathrm{m}$. (B) More GFP-positive cells were found in the subgranular zone of the hippocampus of $\operatorname{Tg}\left(\right.$ Nes-EGFP)33Enik/J; $T b \times 1^{+/+}$mice than in $\operatorname{Tg}\left(\right.$ Nes-EGFP)33Enik/J; $T b \times 1^{+/-}$mice (genotype, $F(1,9)=9.209, P=0.014 ;$ A-P position, $F(12,108)=16.92, P<0.0001$; genotype $\times$ A-P position, $\mathrm{F}(12,108)=1.119, P=0.353)$. For clarity, data are presented as means $\pm \mathrm{SEM}$ but were analyzed using a generalized linear regression model. $\operatorname{Tg}\left(\right.$ Nes-EGFP)33Enik/J; $\operatorname{Tb} \times 1^{+/+}$mice, $N=6 ; \operatorname{Tg}($ NesEGFP)33Enik/J;Tbx1 ${ }^{+/-}$mice, $N=5$. In total, 13 sections were counted per animal. 

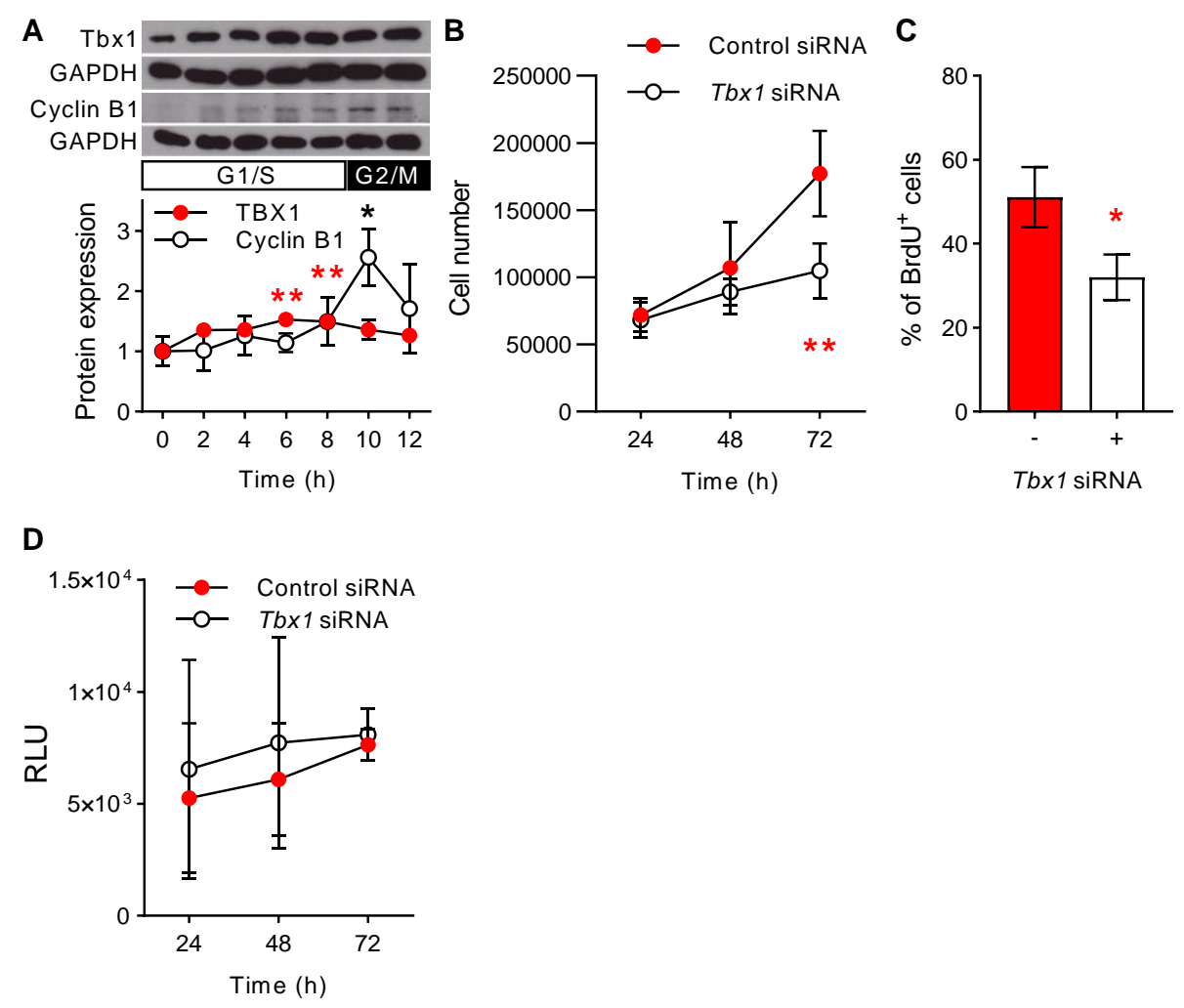

Fig. 2. Effect of Tbx1 deficiency on neonatal neural stem/progenitor cells. (A) TBX1 significantly increased at 6 and $8 \mathrm{~h}$, compared to $0 \mathrm{~h}\left({ }^{* *} P<0.05\right)$. Cyclin B1 expression levels peaked at $10 \mathrm{~h}$, compared to $0 \mathrm{~h}\left({ }^{*} P<0.05\right)$. Protein expression levels were calculated by dividing the intensity of TBX1 or cyclin B1 signals by that of GAPDH. TBX1; $N=6$ per time point, except for $N=4$ at $12 \mathrm{~h}$. Cyclin B1; $N=$ 6 per time point, except for $N=5$ at $6 \mathrm{~h}$ and $N=4$ at $12 \mathrm{~h}$ ). (B) Tbx1 siRNA blunted neonatal stem/progenitor cell proliferation at $72 \mathrm{~h}(* * P=0.001) . N=4,5$, and 7 at 24,48 , and $72 \mathrm{~h}$, respectively, for Control siRNA and Tbx1 siRNA. (C) Tbx1 siRNA significantly reduced BrdU-positive cell number $\left({ }^{*} P=\right.$ 0.04; Control siRNA $N=9, T b \times 1$ siRNA $N=9$ ). (D) Tbx1 siRNA did not alter the apoptosis rate of neonatal stem/progenitor cells, as determined by the RealTime-Glo Annexin $V$ assay $(P>0.05$ for each time point). RLU, relative light unit. Control siRNA $N=9$ and $T b x 1$ siRNA $N=9$ for each time point. 

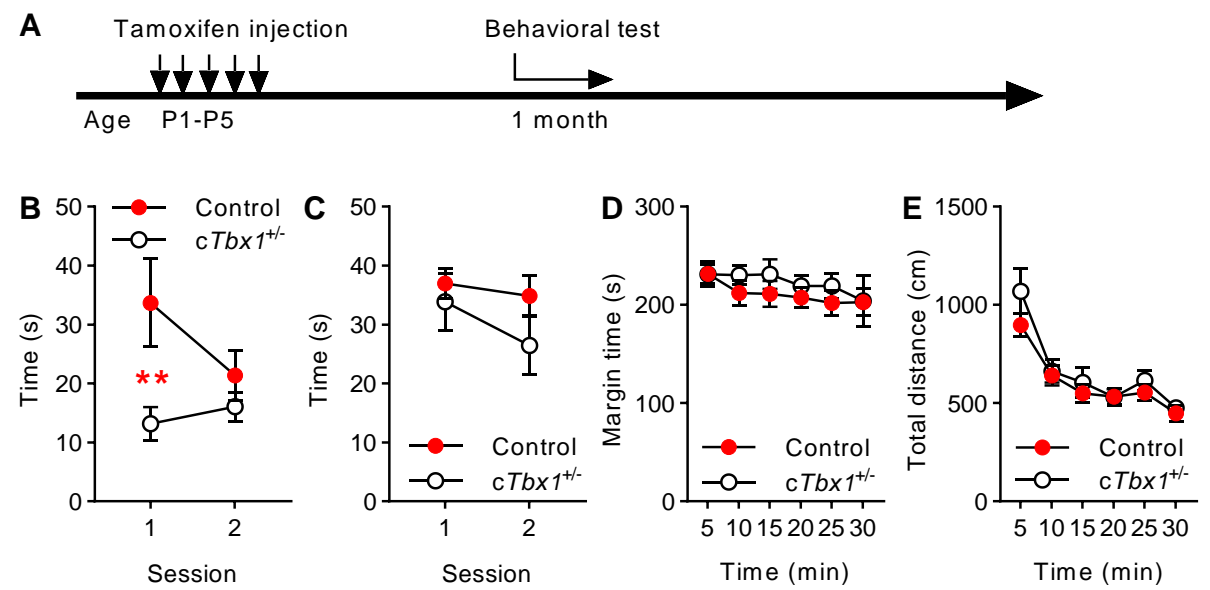

$\mathbf{F}$

Tamoxifen injection

Behavioral test
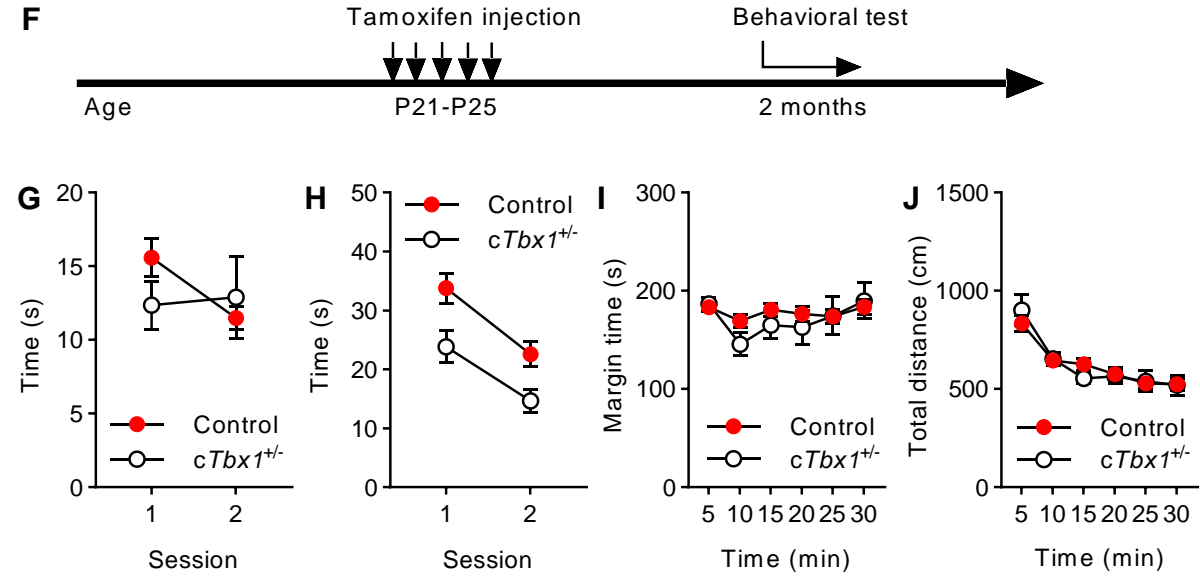

Fig. 3. Behavioral effect of Tbx1 deletion initiated during neonatal and postnatal periods.

Tamoxifen ( 0 and $83.5 \mathrm{mg} / \mathrm{kg}$ body weight, i.p.) was given to lactating mothers of nestinCreERT;Tbx $1^{\text {flox/+ }}$ pups from P1 to P5. Behavioral assays started 1 month later $(\mathbf{A})$ for social approach (B) (Control, $N=19 ; c T b \times 1^{+/}, N=12$ ), novel object approach (C) (Control, $N=$ 23; $\left.c T b \times 1^{+/-}, N=12\right)$, thigmotaxis (D) (Control, $\left.N=14 ; c T b \times 1^{+/-}, N=6\right)$, and motor activity (E) (Control, $\left.N=14 ; c T b \times 1^{+/}, N=6\right) . c T b \times 1^{+/-}$mice showed lower levels of social approach at session 1 than control mice did $(P=0.007)$. The groups did not differ in any other tested behavior $(P>0.05)$. Another set of mice were given tamoxifen ( 0 and $180 \mathrm{mg} / \mathrm{kg}$ body weight, i.p.) from P21 to P25 and were tested 1 month later (F) for social approach (G) (Control, $N=35$; $\left.c T b \times 1^{+-}, N=11\right)$, novel object approach (H) (Control, $\left.N=33 ; c T b \times 1^{+-}, N=12\right)$, thigmotaxis (I) Control, $N=40 ; c T b \times 1^{+/-}, N=12$ ), and motor activity (J) (Control, $N=40 ; c T b \times 1^{+/-}, N=12$ ). 
bioRxiv preprint doi: https://doi.org/10.1101/2021.11.12.468452; this version posted November 13, 2021. The copyright holder for this preprint (which was not certified by peer review) is the author/funder. All rights reserved. No reuse allowed without permission.

$c T b \times 1^{+/-}$mice showed lower levels of novel object approach equally at sessions 1 and 2 compared to control mice (genotype, $F(1,43)=5.151, P=0.028$; genotype $\times$ session, $F(1,43)=$ $0.635, P=0.43)$. The groups did not differ in any other tested behavior $(P>0.05)$. 

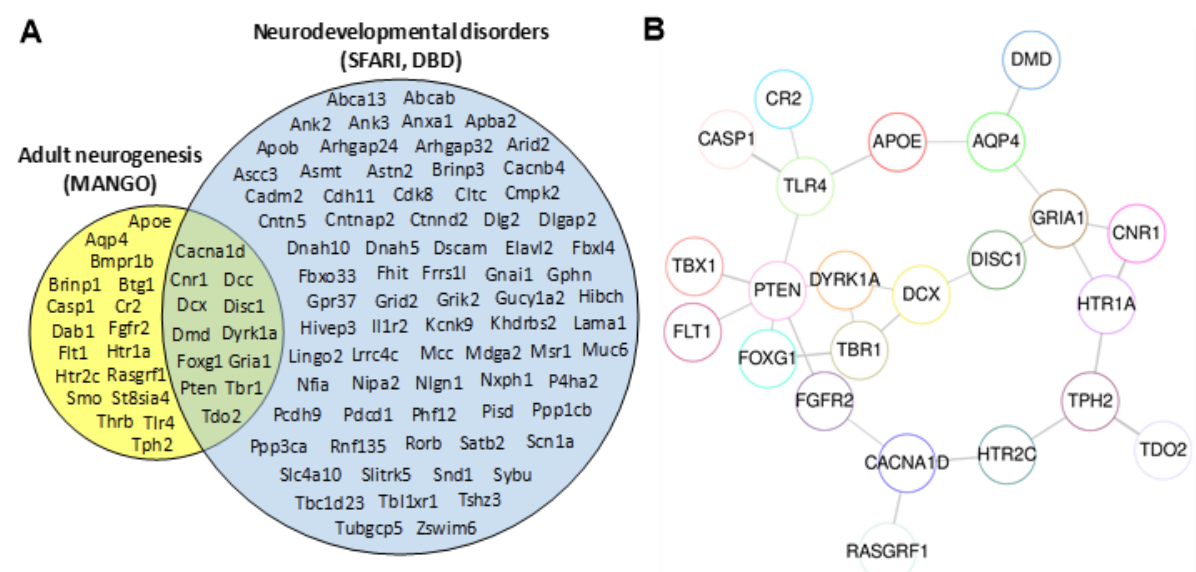

Fig. 4. Genes bound by TBX1 that are implicated in adult neurogenesis and

neurodevelopmental disorders. (A) The following gene databases were used:

(http://mango.adult-neurogenesis.de/documents/annotations?show=20\&expression=true;

MANGO, left yellow circle). Autism and other neurodevelopmental disorders (SFARI,

https://gene.sfari.org; DBD, https://dbd.geisingeradmi.org/) (Right, pale blue circle). (B)

Potential interactions among the target genes of TBX1 are shown, based on Search Tool for

Retrieval of Interacting Genes/Proteins (STRING v11) analysis of MANGO-listed genes (see

yellow circle in A) (41). 
A
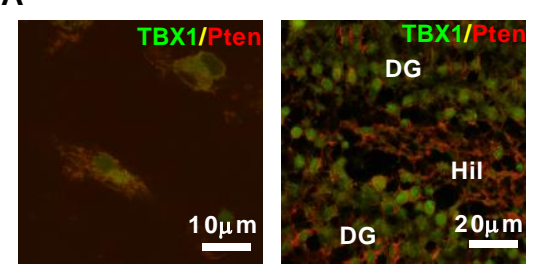

D

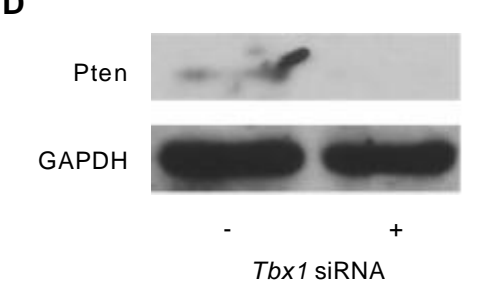

E

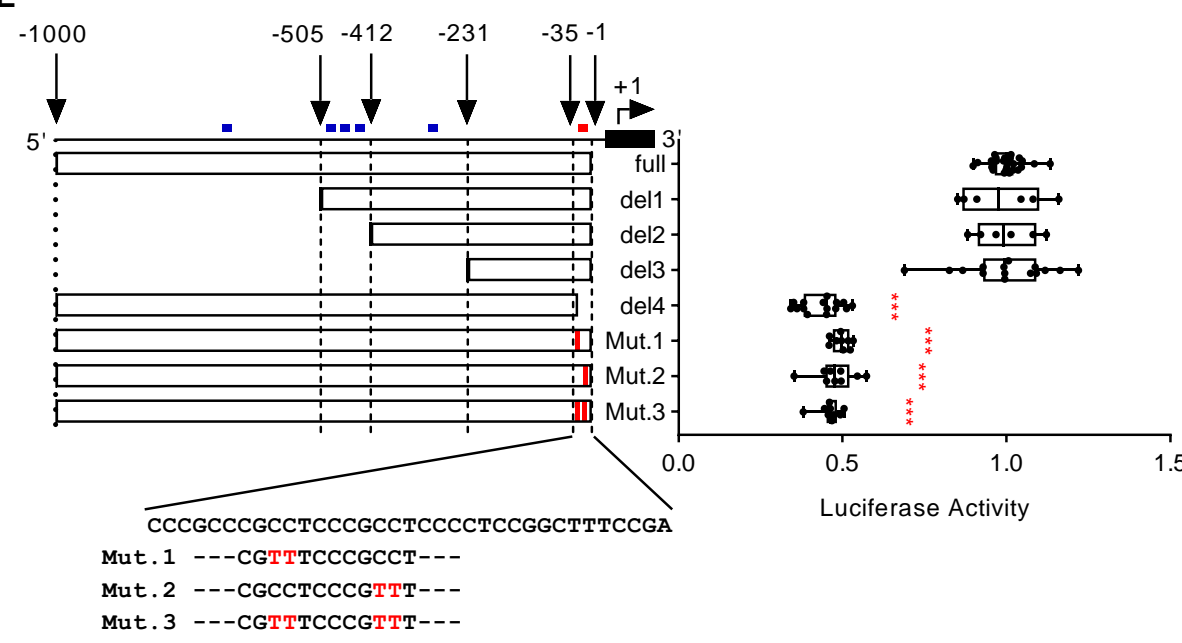

B

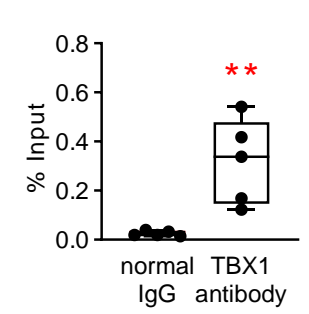

C

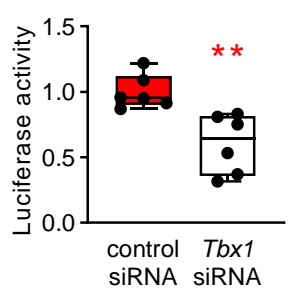

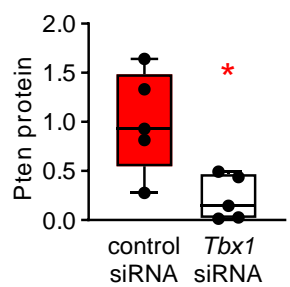

Fig. 5. Transcriptional regulation of Pten by TBX1 in neonatal stem/progenitor cells. (A) TBX1 expression in PTEN-positive stem/progenitor cells in vitro (left) and granule cells in the mouse hippocampus (right). (B) ChIP-PCR. TBX1 binds to the Pten promoter. Input, preimmunoprecipitated diluted solution was the control $(P=0.008 ; N=5$ per group $)$. \%input $=2 \%$ $\times 2$ (C[T] 2\%Input Sample - C[T] IP Sample). (C) Tbx1 siRNA significantly reduced transcriptional activity of the $1 \mathrm{~kb}$ Pten promoter ( $P=0.002 ; N=6$ per group) (see Fig. S1B for Tbx1 siRNA efficacy). (D) Tbx1 siRNA significantly reduced PTEN protein in P0 neural progenitor cells ( $P=$ $0.0016 ; N=5$ per group). (E) Deletion and mutation positions within the $1 \mathrm{~kb}$ Pten promoter (left); their effects on luciferase activity (right). Deletion and mutation of the proximal $35 \mathrm{bp}$ reduced Pten promoter activity (***P<0.001; Mann-Whitney test). T-box-binding consensus 
sequence (blue dots): GTGTTATGACACGTGCAAGTGTGAGTGCGA (42); T-box-binding-like sequence (red dot): CCGCCTCCCGCCT (29). Full, $N=27$; Del.1, $N=6$; Del. 2, $N=6$; Del. 3, $N=15$;

Del. 4, $N=15 ;$ Mut.1, $N=9$; Mut. 2, $N=9$; Mut. 3, $N=9$. 